\title{
Critical Care Nurses' Knowledge and Practices on Ventilator- Associated Pneumonia
}

\author{
Nurul Aqilah Bt Selamat ${ }^{1}$, Khin Thandar Aung ${ }^{1^{*}}$ and May Khin Soe ${ }^{2}$ \\ ${ }^{1}$ Faculty of Nursing, International Islamic University, Malaysia \\ ${ }^{2}$ Faculty of Pharmacy, International Islamic University, Malaysia
}

*Corresponding author: Khin Thandar Aung, MNSC, BNSc, Lecturer, Critical Care Nursing Department, Faculty of Nursing, International Islamic University, Malaysia, Tel: +6012-967-7943

\begin{abstract}
Background: Ventilator-associated pneumonia (VAP) is common in critical care and can happen within 48-72 hours in intubated ventilated patients. Ventilator-associated pneumonia is a condition of high rates of morbidity, mortality, length of stay and hospital costs for ventilated patients. The prevention of (VAP), known as a hospital-acquired infection, is a major clinical challenge such that critical care nurses need to understand the risk factors for developing VAP. And also, nurses are the best position to put their known evidence-based knowledge into practice in preventing (VAP).

Aim: The aim of this study is to assess the knowledge and practices of critical care nurses regarding VAP in selected ICU wards of a tertiary care hospital in Kuantan, Malaysia. Additionally, the study sought to investigate the association between their socio-demographic data and nurses' knowledge and practice of VAP guidelines.
\end{abstract}

Methods/materials: A descriptive cross-sectional survey was conducted on 175 critical care nurses at two hospitals in Kuantan, Malaysia. A Simple random sampling method was used to recruit participants. The tool for data collection was a well-developed questionnaire adapted from two articles 'Critical care nurses' knowledge of evidencebased guidelines for preventing associated Pneumonia: An evaluation questionnaire" (Labeau, et al., 2007) and "Malaysian patient safety goal, nurses' roles and responsibilities, first edition, 2015. Data were analyzed using descriptive statistics and inferential statistics (KruskalWallis test)

Result: The response rate of this study was $77.14 \%$ and the nurses who were working less than 6 months or newly recruit, on maternity leave and further study, not willing to participate and answered the incomplete questionnaire were excluded. The mean score for knowledge of critical care nurses was $6.75 / 8$ and the mean score for the practice of critical care nurses was $48.45 / 50$. The years of experiences of nurses and type of critical care unit were significantly associated with their knowledge and practice on VAP $(p<0.05)$.

Conclusion: Nurses are having good knowledge and practice regarding ventilator-associated pneumonia at Kuantan, Malaysia. The critical care nurses need to be encouraged their knowledge translates into practice toward prevention and care for VAP patients. In addition, one's own institution should monitor regularly, the adherence to the VAP guidelines by the critical care nurses. Thus, the own institution's monitoring and the adherence of the nurses can help in reducing the incidence rate of VAP and promote quality ventilator care for intubated ventilated patients.

\section{Keywords}

Knowledge, Practice, Critical care nurses, Ventilatorassociated pneumonia

\section{Introduction}

Pneumonia associated mechanical ventilation commonly happens within 48-72 hours when the patient was intubated and started a mechanical ventilator [1]. Generally, all countries need to reduce the ventilatorassociated pneumonia (VAP) prevalence in line with reducing the rate of hospital-acquired infections. The cases of VAP not only happened in developed countries such as in the United Sates but also in developing countries like Malaysia. Centre for Disease Control and Prevention has reported that a mean (VAP) was 3.6 cases/1000 ventilator days in the ICU around the United States, whereas 10 to 41.7 cases/1000 ventilator days

Citation: Selamat NAB, Aung KT, Soe MK (2021) Critical Care Nurses' Knowledge and Practices on Ventilator-Associated Pneumonia. Int Arch Nurs Health Care 7:163. doi.org/10.23937/24695823/1510163

Accepted: July 24, 2021: Published: July 26, 2021

Copyright: (C) 2021 Selamat NAB, et al. This is an open-access article distributed under the terms of the Creative Commons Attribution License, which permits unrestricted use, distribution, and reproduction in any medium, provided the original author and source are credited. 
happened in a developing country [2]. In Malaysia, The Malaysia Registry of Intensive Care report (2016) specified that the VAP rate was 2.4 per 1000 ventilator days in the year 2015 [3]. Thus, preventive guidelines for (VAP) should adhere to achieve the goal of reducing the rate of ventilator-associated pneumonia. There are various preventive measures for (VAP) such as practicing good hand hygiene before touching patients and ventilator, maintaining good oral hygiene of the patient. Moreover, limiting the duration of mechanical ventilation, reducing colonization by practicing oral care using chlorohexidine, maintaining the patients in a semirecumbent position to prevent aspiration, and ensuring a sufficient cuff pressure are also needed in ventilator care [4]. The studies regarding the intervention of VAP, had been done, however, a study on assessing knowledge and practice of critical care nurses regarding VAP guidelines in Malaysia are still inadequate and need to be explored $[4,5]$. Considering the significance of critical care nurses as a front liner providing ventilator care and preventing pneumonia, there is needed to investigate the knowledge and practice of critical care nurses on VAP and also determine the association between socio-demographic data with knowledge and practices of critical care nurses on VAP.

\section{Materials and Methods}

A cross-sectional descriptive study was conducted among critical care nurses from Hospital Tengku Ampuan Afzan (HTAA) and International Islamic University Malaysia Medical Campus (IIUMMC), Kuantan, Pahang State, Malaysia. Critical care nurses from Intensive Care Unit (ICU), Cardiovascular Intensive Care Unit (CCU), Paediatric Intensive Care Unit (PICU) and Neonatal Intensive Care Unit (NICU) were selected as participants using a simple random sampling method and the total was 175 participants. Those who were working less than 6 six months or newly recruit, on maternity leave and further study, not willing to participate and answered the incomplete questionnaire were excluded. Therefore, the response rate of this study was $77.14 \%(n=135)$.

A well-developed questionnaire adapted from an article 'Critical care nurses' knowledge of evidencebased guidelines for preventing associated pneumonia: An evaluation questionnaire" [6]. (Labeau, et al., 2007) and "Malaysian patient safety goal, nurses' roles and responsibilities first edition 2015" were used as research instruments in this study [7]. The questionnaires were presented to three experts including doctors and nursing lecturers for content validity. To achieve face validity, ten critical care nurses were asked if they have any comments regarding the layout of the questionnaire or items that they do not understand. A pilot test was also conducted to pretest the questionnaire which resulted Cronbach's alpha is $\geq 0.7$. The questionnaire included

Table 1: Socio-demographic data of participants.

\begin{tabular}{|c|c|c|c|c|}
\hline$N=135$ & Variables & Mean & Frequency & Percentage \\
\hline \multirow[t]{5}{*}{ Age } & & 33.81 & & \\
\hline & $21-30$ & & 50 & 37.0 \\
\hline & $31-40$ & & 69 & 51.1 \\
\hline & $41-50$ & & 15 & 11.1 \\
\hline & $51-60$ & & 1 & 0.7 \\
\hline \multirow[t]{2}{*}{ Gender } & Male & & 4 & 3.0 \\
\hline & Female & & 131 & 97.0 \\
\hline \multirow[t]{4}{*}{ Year of experience } & 6 months - 2 years & & 22 & 16.3 \\
\hline & $3-5$ years & & 33 & 24.2 \\
\hline & $6-10$ years & & 41 & 30.4 \\
\hline & $>10$ years & & 39 & 28.9 \\
\hline \multirow[t]{5}{*}{ Level of education } & Diploma & & 73 & 54.1 \\
\hline & Post Basic (Speciality) & & 58 & 43.0 \\
\hline & Bachelor Degree & & 3 & 2.2 \\
\hline & Master Degree & & 1 & 0.7 \\
\hline & $\mathrm{PhD}$ & & 0 & 0 \\
\hline \multirow[t]{4}{*}{ Type of critical care } & (ICU) & & 69 & 51.1 \\
\hline & (CCU) & & 8 & 5.9 \\
\hline & (PICU) & & 34 & 25.2 \\
\hline & $(\mathrm{NICU})$ & & 24 & 17.8 \\
\hline \multirow[t]{3}{*}{ Number of workshop attends } & Never Attend & & 67 & 49.6 \\
\hline & 1 time & & 51 & 37.8 \\
\hline & $>2$ times & & 17 & 12.5 \\
\hline
\end{tabular}


the demographic data, knowledge, and practice on VAP guidelines.

The researcher obtained ethical approval from Medical Research Ethics Committee (MREC) and Kulliyyah Nursing Post Graduate Research Committee (KNPGRC). The purpose of this study was explained to participants and ensured voluntary participation with informed consent. All answered questionnaire were returned in a sealed envelope, and all information was kept as anonymous. Statistical analysis program (SPSS) version 21 was used and data were analyzed using frequencies and percentages as well as inferential analysis using Kruskal-Wallis test. The $p$-value $<0.05$ was set as $<0.05$ to determine statistical significance.

\section{Results}

The response rate of this study was 77.14\%. ( $n=$ 135). Among the 135 respondents, comprising four males (3.0\%) and 131 females (97.0\%). The majority of the age group of the critical care nurses were 31-40 years, with a mean age of 33.81. Out of the four critical units, there were 69 nurses from Intensive Care Unit (ICU) (51.1\%), eight nurses from Cardiovascular Care Unit (CCU) (5.9\%), 34 nurses from Paediatric Intensive Care Unit (PICU) (25.2\%), and 24 nurses from Neonatal Intensive Care Unit (NICU) (17.8\%). In terms of year of experience in the critical care unit, $6-10$ year is the highest with a total of 41 nurses (30.4\%), 6 months to-2 years of experiences with 22 nurses (16.3\%), 3-5 year of experience with 33 nurses (24.4\%), and more than 10 years of experience with 39 nurses (28.9\%) as shown in Table 1. Regarding the education level, the participants had five different levels of education which are diploma level consisted of $73(54.1 \%)$, diploma with post basic (speciality) were 58 nurses (43.0\%), bachelor level were 3 nurses (2.2\%), master level were one nurse $(0.7 \%)$, and no PhD level as also shown in Table 1.

Concerning the knowledge on VAP among participants, the result showed $74.8 \%(n=101)$ of the respondents recognized that the oral route is the recommended way for intubation, while $97.8 \%$ ( $n=$ 132) that ventilator circuits should be changed every new patient (Table 2). Additional findings were most of the participants reported a strong knowledge of how to provide ventilator care such as type of humidifier, closed suction systems, and 30-45-degree patient's bed elevation is as the best position to prevent VAP.

Regarding the practice on VAP, out of 135 respondents, majority of participants $97.8 \%(n=132)$ practiced hand washing before and after touching the patients. 93.3\% ( $n=126)$ always follow the ICU protocol in enteral feeding. More than half that is $65.2 \%(n=$ 88) maintained oral hygiene with $0.2 \%$ chlorohexidine

Table 2: Knowledge of (VAP) among participants.

\begin{tabular}{|c|c|c|c|c|c|}
\hline \multicolumn{2}{|c|}{ Knowledge of Ventilator Associated Pneumonia } & Frequency (n) & \multicolumn{2}{|c|}{ Percentage (\%) } & Mean \\
\hline \multicolumn{2}{|c|}{ Oral route recommended for endotracheal intubation } & 101 & \multicolumn{2}{|l|}{74.8} & 1.67 \\
\hline \multicolumn{2}{|c|}{ Ventilator circuit should be changing every new patient } & 132 & \multicolumn{2}{|l|}{97.8} & 1.97 \\
\hline \multicolumn{2}{|c|}{ Heat and moisture recommended type of humidifier } & 90 & \multicolumn{2}{|l|}{66.7} & 1.64 \\
\hline \multicolumn{2}{|c|}{ Closed suction system recommended in preventing VAP } & 126 & \multicolumn{2}{|l|}{93.3} & 1.87 \\
\hline \multicolumn{2}{|c|}{ Closed suction system must be changed every new patient } & 108 & \multicolumn{2}{|l|}{80.0} & 1.79 \\
\hline \multicolumn{2}{|c|}{$30-45$ degree head elevation is best position to prevent VAP } & 123 & \multicolumn{2}{|l|}{91.1} & 1.84 \\
\hline Overall & Total Participants & \multicolumn{2}{|l|}{ Mean Score } & \multicolumn{2}{|c|}{ Standard Deviation } \\
\hline Knowledge score & 135 & \multicolumn{2}{|l|}{6.75} & \multicolumn{2}{|l|}{1.042} \\
\hline
\end{tabular}

Table 3: Practice of (VAP) among participants.

\begin{tabular}{|c|c|c|c|}
\hline Practice of Ventilator Associated Pneumonia & Frequency (n) & $\begin{array}{l}\text { Percentage } \\
(\%)\end{array}$ & Mean \\
\hline Wash hand before and after touching another patient & 132 & 97.8 & 4.98 \\
\hline Follow ICU protocol in enteral feeding & 126 & 93.3 & 4.92 \\
\hline Maintain oral hygiene with $0.2 \%$ chlorohexidine or sterile water & 88 & 65.2 & 4.61 \\
\hline Perform closed suction system in suctioning & 126 & 93.3 & 4.93 \\
\hline Maintain 30-45-degree elevation of the head of bed & 115 & 85.2 & 4.84 \\
\hline Check tracheostomy tube every shift & 102 & 75.6 & 4.71 \\
\hline Perform barrier nursing for infected patients & 129 & 95.6 & 4.96 \\
\hline Maintain pressure of the pilot balloon of the endotracheal tube at $25-30 \mathrm{cmH}_{2} \mathrm{O}$ & 107 & 79.3 & 4.67 \\
\hline Aspirate the secretions from endotracheal tube and mouth before extubating & 130 & 96.3 & 4.96 \\
\hline Overall & $\begin{array}{l}\text { Total } \\
\text { Participants }\end{array}$ & Mean Score & $\begin{array}{l}\text { Standard } \\
\text { Deviation }\end{array}$ \\
\hline Practice score & 135 & 48.45 & 1.911 \\
\hline
\end{tabular}


or sterile water. Most of the participants performed closed suction system in suctioning by $93.3 \%(n=126)$, and $85.2 \%(n=115)$ maintained $30-45$-degree elevation of the patient's bed to prevent aspiration pneumonia. Furthermore, $75.6 \%(n=102)$ respondents checked the tracheostomy tube in every shift and $79.3 \%$ ( $n=$ 107) maintained pressure of the pilot balloon of the endotracheal tube. It was shown in Table 3.

A Kruskal-Wallis test was used to reveal a statistically significant difference in knowledge score and practice across year of experience and type of critical care unit. There was significantly difference between knowledge score and year of experience with the $\chi^{2}(3, n=135)$ $=12.674, p=0.005$ and, also significant difference between knowledge score and types of critical care unit showed $\chi^{2}(3, n=135)=7.974, p=0.047$. Besides that, the significance different between practice score and year of experience was $\chi^{2}(3, n=135)=8.953, p=0.030$ and significance different between practice score and types of critical unit was $\chi^{2}(3, n=135)=16.132, p=$ 0.001 were shown in Table 4. The result indicated that year of experience and types of critical care unit were related to knowledge and practice of critical care nurses regarding ventilator-associated pneumonia.

\section{Discussion}

Findings showed that half of critical care nurses had a good level of knowledge on VAP. As sequences, critical care nurses increased their action in precautions to prevent VAP. However, there was a previous study among Belgium nurses revealed that critical care nurses have an average level of knowledge regarding VAP [6]. Thus, this previous study was quite different with this study. On the other hand, Malaysia critical care nurses had already exposed job training regarding VAP. Besides, the overall result for practice level among critical care nurses revealed that a majority of participants have a satisfactory level of practice on VAP care bundles. However, one study showed that there was no satisfactory level of practice in Spanish [5].

In this study, the results showed that there was a significant difference between years of experience and knowledge on VAP among participants. And also, found that nurses' experience influenced on their level of knowledge. This finding was similar with previous study in Flemish, Belgium in which there was a significant difference between the knowledge of nurses on VAP and their working experience [6]. In addition, in this study, there was also a significant difference between year of experience and practice on VAP guidelines. It meaned that the experiences of nurses also impacted to their practice. In contrast, Kalyan G, Bibi R, Kaur R, et al. found among Indian nurses that they possessed good to average knowledge scores on VAP. However, their practices were not associated with their knowledge scores and there were needed to implement new strategies to improve in practicing VAP guidelines [8]. Apart from that, the types of critical care unit were also a significant difference on nurses' knowledge and practice on VAP. Hence, different critical care unit provided different knowledge and practice on VAP guidelines.

\section{Limitation}

This study was done only two hospitals in Pahang State, East Coast region of Malaysia due to time constraint, which cannot be generalized for the whole nurses' population in Malaysia. As suggestion, a future study should be done in other states of Malaysia as well. Regarding the research instruments, this study was only used the theoretical knowledge and practice questionnaires based on VAP evidenced based guidelines. Therefore, there was suggested to use an observation checklist in measuring the practice of nurses for future study.

\section{Conclusion}

As conclusion, this study achieved the study's aim and found that Malaysian nurses had good knowledge and practice regarding VAP. However, it is very important for the hospital to monitor regularly among the health care personnel in regards to the adherence on VAP guidelines for the prevention of hospital-acquired

Table 4: The significant different between socio-demographic characteristics and knowledge, practice on (VAP) among participants.

\begin{tabular}{|l|l|l|l|l|}
\hline Socio-demographic data & Ventilator Associated & \multicolumn{2}{l|}{ Kruskal-Wallis Test } \\
\cline { 2 - 4 } & Pneumonia & Chi square & df & Asymp. Sig \\
\hline Age & Knowledge score & 3.290 & 3 & 0.349 \\
\hline Year of Experience & Knowledge score & 12.674 & 3 & $\mathbf{0 . 0 0 5}$ \\
\hline Level of Education & Knowledge score & 7.204 & 3 & 0.066 \\
\hline Type of critical care unit & Knowledge score & 7.947 & 3 & $\mathbf{0 . 0 4 7}$ \\
\hline Workshop attend & Knowledge score & 2.308 & 2 & 0.315 \\
\hline Age & Practice score & 5.631 & 3 & 0.131 \\
\hline Year of Experience & Practice score & $\mathbf{8 . 9 5 3}$ & 3 & $\mathbf{0 . 0 3 0}$ \\
\hline Level of Education & Practice score & 3.585 & 3 & 0.310 \\
\hline Type of critical care unit & Practice score & $\mathbf{1 6 . 1 3 2}$ & 3 & $\mathbf{0 . 0 0 1}$ \\
\hline Workshop attend & Practice score & 0.926 & 2 & 0.629 \\
\hline
\end{tabular}


infections. Future research related to VAP guidelines adherence training are needed among critical care nurses for professional development. Overall, this study provided some benefits for patients, nurses as well as health care institutions in managing to reduce hospitalacquired infections and promote the well-being of intubated ventilated patients in ventilator care.

\section{Acknowledgements}

The authors gratefully acknowledge the authorities Hospital Tengku Ampuan Afzan (HTAA) and International Islamic University Malaysia Medical Centre (IIUMMC) for their constant support. We would also like to thank the critical care nurses who have actively participated in the study.

\section{Conflict of Interest}

The authors declare no conflicts of interest.

\section{References}

1. Subramanian $\mathrm{P}$, Choy $\mathrm{KL}$, Gobal SV, Mansor $\mathrm{M}, \mathrm{Ng} \mathrm{KH}$ (2013) Impact of education on ventilator-associated pneumonia in the intensive care unit. Singapore Med J 54: 281-284.
2. American Thoracic Society, Infectious Diseases Society of America (2005) Guidelines for the management of adults with hospital-acquired, ventilator-associated, and healthcare-associated pneumonia. Am J Respir Crit Care Med 171: 388-416.

3. Geok JTM, Ling TL, Cheng TC, Har LC, Ismail NI (2016) Malaysian Registry of Intensive Care Report for 2015.

4. Lambert ML, Palomar M, Agodi A, Hiesmayr M, Lepape A, et al. (2013) Prevention of ventilator-associated pneumonia in intensive care units: An international online survey. Antimicrob Resist Infect Control 2: 9.

5. Rello J, Afonso E, Lisboa T, Ricart M, Balsera B, et al. (2013) A care bundle approach for prevention of ventilatorassociated pneumonia. Clin Microbiol Infect 19: 363-369.

6. Labeau S, Vandijck DM, Claes B, Aken PV, Blot SI (2007) Critical care nurse's knowledge of evidence- based guidelines for preventing ventilator-associated pneumonia: An evaluation questionnaire. Am J Crit Care 16: 371-377.

7. (2015) Malaysian patient safety goals, nurses roles and responsibilities. ( $1^{\text {st }}$ edn), Ministry of Health, Malaysia.

8. Kalyan G, Bibi R, Kaur R, Bhatti R, Kumari R, et al. (2020) Knowledge and practices of intensive care unit nurses related to prevention of ventilator associated pneumonia in selected intensive care units of a tertiary care centre, India. Iran J Nurs Midwifery Res 25: 369-375. 